\title{
Potential and Sobolev Spaces Related to Symmetrized Jacobi Expansions ${ }^{\star}$
}

Bartosz LANGOWSKI

Wydziat Matematyki, Politechnika Wroctawska, Wyb. Wyspiańskiego 27, 50-370 Wroctaw, Poland

E-mail: bartosz.langowski@pwr.edu.pl

Received May 08, 2015, in final form September 10, 2015; Published online September 12, 2015 http://dx.doi.org/10.3842/SIGMA.2015.073

\begin{abstract}
We apply a symmetrization procedure to the setting of Jacobi expansions and study potential spaces in the resulting situation. We prove that the potential spaces of integer orders are isomorphic to suitably defined Sobolev spaces. Among further results, we obtain a fractional square function characterization, structural theorems and Sobolev type embedding theorems for these potential spaces.
\end{abstract}

Key words: Jacobi expansion; potential space; Sobolev space; fractional square function

2010 Mathematics Subject Classification: 42C10; 42C05; 42C20

\section{Introduction}

This article is motivated by the recent results of Nowak and Stempak [25] and the author's papers $[16,17]$. In [16] we investigated Sobolev and potential spaces related to discrete Jacobi function expansions. The main achievement of [16] is a suitable definition of the SobolevJacobi spaces so that they are isomorphic with the potential spaces with appropriately chosen parameters. The article [16] is a continuation and extension of a similar study conducted in the setting of ultraspherical expansions by Betancor et al. [4]. The other author's paper [17] contains further investigations of the Jacobi potential spaces. The most important outcome of [17] is a characterization of the potential spaces by means of suitably defined fractional square functions. The research in [17] was inspired by another paper of Betancor et al. [5], in which a general technique of using square functions in analysis of potential spaces associated with discrete and continuous orthogonal expansions was developed.

On the other hand, in [25] Nowak and Stempak proposed a symmetrization procedure in a context of general discrete orthogonal expansions related to a second-order differential operator $L$, a 'Laplacian'. This procedure, combined with a unified conjugacy scheme established in an earlier article by the same authors [24], allows one to associate, via a suitable embedding, a differential-difference 'Laplacian' $\mathbb{L}$ with the initially given orthogonal system of eigenfunctions of $L$ so that the resulting extended conjugacy scheme has the natural classical shape. In particular, the related 'partial derivatives' decomposing $\mathbb{L}$ are skew-symmetric in an appropriate $L^{2}$ space and they commute with Riesz transforms and conjugate Poisson integrals. Thus the symmetrization procedure overcomes the main inconvenience of the theory postulated in [24], that is the lack of symmetry in the principal objects and relations resulting in essential deviations of the theory from the classical shape. The price is, however, that the 'Laplacian' $\mathbb{L}$ and the associated 'derivatives' are not differential, but differential-difference operators. It was shown in [25] that the symmetrization is supported by a good $L^{2}$ theory. Moreover, in [15] the author

\footnotetext{
${ }^{\star}$ This paper is a contribution to the Special Issue on Orthogonal Polynomials, Special Functions and Applications. The full collection is available at http://www.emis.de/journals/SIGMA/OPSFA2015.html
} 
verified that further support comes from the $L^{p}$ theory, at least when the Jacobi polynomial context is considered.

In the present paper we apply the above mentioned symmetrization procedure to the setting of Jacobi function expansions considered in $[16,17]$. Then we define and study the associated potential spaces and Sobolev spaces. As the main results, we establish an isomorphism between these spaces (Theorem 3.3) and characterize the potential spaces by means of suitably defined fractional square functions (Theorems 4.2 and 4.3). Among further results, we prove structural and embedding theorems for the potential spaces, in particular we obtain a counterpart of the classical Sobolev embedding theorem in the Jacobi setting (see Theorems 5.1 and 5.3). All of this extends the results from $[16,17]$ to the symmetrized situation.

The general strategy we use to prove the results in the symmetrized setting relies on two steps. In the first step we exploit symmetries of the operators under consideration in order to reduce the analysis essentially to the initial non-symmetrized case. Then the second step consists in taking advantage of the results already existing in the literature, mostly from author's previous articles $[16,17]$. Even though the general line of reasoning is relatively easy, some details occur to be rather technical and complex.

An important aspect, and in fact also a partial motivation of our research, is the suggestion from [25, Section 5] that the symmetrization could have a significant impact on developing the theory of Sobolev spaces related to orthogonal expansions. This concerns, in particular, higher-order 'derivatives' leading to appropriate Sobolev spaces. It turns out, however, that in our symmetrized framework the relevant higher-order 'derivatives' are not constructed from the first-order 'derivative' (see Proposition 3.4), as one would perhaps expect after reading the optimistic comments in [24, Section 5]. Thus these derivatives are even more exotic than the variable index derivatives that are suitable in the initial non-symmetrized Jacobi setting. So it seems that the symmetrization brings no improvement in dealing with Sobolev spaces, at least in the Jacobi setting considered. This makes a noteworthy contrast to the conjugacy scheme which benefits a lot from the symmetrization.

Sobolev and potential spaces related to different classical orthogonal expansions were investigated in recent years by various authors, see, e.g., $[2,3,4,5,6,7,13,16,17,26]$. On the other hand, harmonic analysis in several frameworks of Jacobi expansions was in the last decade studied in $[2,3,8,9,15,16,17,19,20,21,22,23,28]$ (see also [11, 12]), among others. Our present work contributes to both of these lines of research.

An interesting study of variable exponent Sobolev spaces for Jacobi expansions is contained in the recent paper by Almeida, Betancor, Castro, Sanabria and Scotto [1]. The results of [1] are related to those in the author's papers $[16,17]$, but were obtained independently. In particular, there is a partial overlap in characterizations of the Jacobi potential spaces via fractional square functions obtained in [17] and [1]. We thank one of the referees for bringing [1] to our attention.

\section{Notation}

Throughout the paper, we use a fairly standard notation with essentially all symbols referring either to the measure space $((-\pi, \pi), d \theta)$ or the restricted space $((0, \pi), d \theta)$. Given a function $f$ on $(-\pi, \pi)$, we denote by $f^{+}$its restriction to the subinterval $(0, \pi)$, and by $f_{\text {even }}$ and $f_{\text {odd }}$ its even and odd parts, respectively,

$$
f_{\text {even }}(\theta)=\frac{f(\theta)+f(-\theta)}{2}, \quad f_{\text {odd }}(\theta)=\frac{f(\theta)-f(-\theta)}{2} .
$$

We let

$$
\left\langle f_{1}, f_{2}\right\rangle=\int_{-\pi}^{\pi} f_{1}(\theta) \overline{f_{2}(\theta)} d \theta, \quad\left\langle h_{1}, h_{2}\right\rangle_{+}=\int_{0}^{\pi} h_{1}(\theta) \overline{h_{2}(\theta)} d \theta
$$


whenever the integrals make sense. For $1 \leq p \leq \infty, p^{\prime}$ denotes its conjugate exponent, $1 / p+$ $1 / p^{\prime}=1$. When writing estimates, we will use the notation $X \lesssim Y$ to indicate that $X \leq C Y$ with a positive constant $C$ independent of significant quantities. We shall write $X \simeq Y$ when simultaneously $X \lesssim Y$ and $Y \lesssim X$.

\section{Preliminaries}

Given parameters $\alpha, \beta>-1$, consider the Jacobi differential operator

$$
L_{\alpha, \beta}=-\frac{d^{2}}{d \theta^{2}}-\frac{1-4 \alpha^{2}}{16 \sin ^{2} \frac{\theta}{2}}-\frac{1-4 \beta^{2}}{16 \cos ^{2} \frac{\theta}{2}}=D_{\alpha, \beta}^{*} D_{\alpha, \beta}+A_{\alpha, \beta}^{2}
$$

here $A_{\alpha, \beta}=(\alpha+\beta+1) / 2$ is a constant, and

$$
D_{\alpha, \beta}=\frac{d}{d \theta}-\frac{2 \alpha+1}{4} \cot \frac{\theta}{2}+\frac{2 \beta+1}{4} \tan \frac{\theta}{2}, \quad D_{\alpha, \beta}^{*}=D_{\alpha, \beta}-2 \frac{d}{d \theta},
$$

are the first-order 'derivative' naturally associated with $L_{\alpha, \beta}$ and its formal adjoint in $L^{2}(0, \pi)$, respectively. It is well known that $L_{\alpha, \beta}$, defined initially on $C_{c}^{2}(0, \pi)$, has a non-negative selfadjoint extension in $L^{2}(0, \pi)$ whose spectral decomposition is discrete and given by the Jacobi functions $\phi_{n}^{\alpha, \beta}, n \geq 0$. The corresponding eigenvalues are $\lambda_{n}^{\alpha, \beta}=\left(n+A_{\alpha, \beta}\right)^{2}$, and the system $\left\{\phi_{n}^{\alpha, \beta}: n \geq 0\right\}$ constitutes an orthonormal basis in $L^{2}(0, \pi)$. Some problems in harmonic analysis related to $L_{\alpha, \beta}$ were investigated recently in $[1,16,17,19,22,28]$.

When $\alpha, \beta \geq-1 / 2$, the functions $\phi_{n}^{\alpha, \beta}$ belong to all $L^{p}(0, \pi), 1 \leq p \leq \infty$. However, if $\alpha<-1 / 2$ or $\beta<-1 / 2$, then $\phi_{n}^{\alpha, \beta}$ are in $L^{p}(0, \pi)$ if and only if $p<-1 / \min (\alpha+1 / 2, \beta+1 / 2)$. This leads to the restriction $p^{\prime}(\alpha, \beta)<p<p(\alpha, \beta)$ for $L^{p}$ mapping properties of various operators associated with $L_{\alpha, \beta}$, where

$$
p(\alpha, \beta):= \begin{cases}\infty, & \alpha, \beta \geq-1 / 2, \\ -1 / \min (\alpha+1 / 2, \beta+1 / 2), & \text { otherwise }\end{cases}
$$

Consequently, many results in harmonic analysis of $L_{\alpha, \beta}$ are restricted to $p \in E(\alpha, \beta)$,

$$
E(\alpha, \beta):=\left(p^{\prime}(\alpha, \beta), p(\alpha, \beta)\right) .
$$

In this work we shall consider the setting related to the larger interval $(-\pi, \pi)$ equipped with Lebesgue measure. An application of the symmetrization procedure from [25] to the context of $L_{\alpha, \beta}$ brings in the following symmetrized Jacobi 'Laplacian' and the associated 'derivative':

$$
\mathbb{L}_{\alpha, \beta}=-\mathbb{D}_{\alpha, \beta}^{2}+A_{\alpha, \beta}^{2},
$$

with

$$
\mathbb{D}_{\alpha, \beta} f=\frac{d f}{d \theta}-\left(\frac{2 \alpha+1}{4} \cot \frac{\theta}{2}-\frac{2 \beta+1}{4} \tan \frac{\theta}{2}\right) \check{f}=D_{\alpha, \beta} f_{\text {even }}-D_{\alpha, \beta}^{*} f_{\text {odd }},
$$

where $\check{f}(\theta)=f(-\theta)$ is the reflection of $f$, and $D_{\alpha, \beta}$ and $D_{\alpha, \beta}^{*}$ are given on $(-\pi, \pi)$ by $(2.1)$. Note that, due to the reflection occurring in $\mathbb{D}_{\alpha, \beta}$, we deal here with a Dunkl type operator. For more details concerning Jacobi-Dunkl operators we refer to [10], see also [14, Section 7].

Also, the following remark is in order. Formally, the space underlying the symmetrized setting is the sum $(-\pi, 0) \cup(0, \pi)$. Nevertheless, often it can (and will) be identified with the interval $(-\pi, \pi)$, since for some aspects of the theory the single point $\theta=0$ is negligible. A typical 
example here are $L^{p}$ inequalities which "do not see" sets of null measure. On the other hand, some objects in the symmetrized situation may not even be properly defined at $\theta=0$ (the latter may in addition depend on the parameters of type), hence this point must be excluded from some considerations like, for instance, continuity or smoothness questions. That is why in what follows several times $(-\pi, \pi) \backslash\{0\}$ appears rather than $(-\pi, \pi)$.

The orthonormal basis in $L^{2}(-\pi, \pi)$ arising from the symmetrization procedure applied to the system of Jacobi functions is

$$
\Phi_{n}^{\alpha, \beta}=\frac{1}{\sqrt{2}} \begin{cases}\phi_{n / 2}^{\alpha, \beta}, & n \text { even }, \\ -\left(\lambda_{(n+1) / 2}^{\alpha, \beta}-\lambda_{0}^{\alpha, \beta}\right)^{-1 / 2} D_{\alpha, \beta} \phi_{(n+1) / 2}^{\alpha, \beta}, & n \text { odd },\end{cases}
$$

where $\phi_{n}^{\alpha, \beta}$ are even extensions (denoted still by the same symbol) to $(-\pi, \pi)$ of the Jacobi functions. More precisely,

$$
\phi_{n}^{\alpha, \beta}(\theta)=c_{n}^{\alpha, \beta} \Psi^{\alpha, \beta}(\theta) P_{n}^{\alpha, \beta}(\cos \theta), \quad \theta \in(-\pi, \pi), \quad n \geq 0,
$$

where $c_{n}^{\alpha, \beta}$ are suitable normalizing constants, $P_{n}^{\alpha, \beta}$ are the classical Jacobi polynomials as defined in Szegő's monograph [29], and

$$
\Psi^{\alpha, \beta}(\theta):=\left|\sin \frac{\theta}{2}\right|^{\alpha+1 / 2}\left(\cos \frac{\theta}{2}\right)^{\beta+1 / 2} .
$$

Observe that $D_{\alpha, \beta} f$ is an odd (even) function if $f$ is even (odd). Consequently, $\Phi_{n}^{\alpha, \beta}$ is even (odd) if and only if $n$ is an even (odd) number. By using [16, formula (5)] we find that

$$
\Phi_{2 n}^{\alpha, \beta}(\theta)=\frac{1}{\sqrt{2}} \phi_{n}^{\alpha, \beta}(\theta), \quad \Phi_{2 n+1}^{\alpha, \beta}(\theta)=\frac{1}{\sqrt{2}} \operatorname{sign}(\theta) \phi_{n}^{\alpha+1, \beta+1}(\theta), \quad n \geq 0 .
$$

Notice that when $\alpha \geq-1 / 2$, all $\Phi_{n}^{\alpha, \beta}, n \geq 0$, are continuous functions on $(-\pi, \pi)$; on the other hand, for $\alpha<-1 / 2$ and $n$ even a singularity at $\theta=0$ occurs. It is a nice coincidence that in our setting $\Phi_{n}^{\alpha, \beta}$ are essentially $\phi_{k}^{\alpha, \beta}$ or $\phi_{k}^{\alpha, \beta}$ with shifted parameters. Roughly speaking, this makes the analysis in the symmetrized situation reducible to the analysis in the initial, nonsymmetrized setting. In general, and even in other Jacobi contexts (see, e.g., [15]), things are more complicated.

According to [25, Lemma 3.5], each $\Phi_{n}^{\alpha, \beta}$ is an eigenfunction of the symmetrized Jacobi operator. More precisely,

$$
\mathbb{L}_{\alpha, \beta} \Phi_{n}^{\alpha, \beta}=\lambda_{\langle n\rangle}^{\alpha, \beta} \Phi_{n}^{\alpha, \beta}, \quad n \geq 0,
$$

where we use the notation $\langle n\rangle=\left\lfloor\frac{n+1}{2}\right\rfloor$ introduced in [25] (here $\lfloor\cdot\rfloor$ denotes the floor function). Thus $\mathbb{L}_{\alpha, \beta}$, considered initially on $C_{c}^{2}((-\pi, \pi) \backslash\{0\})$, has a natural self-adjoint extension to $L^{2}(-\pi, \pi)$, denoted by the same symbol, and given by

$$
\mathbb{L}_{\alpha, \beta} f=\sum_{n=0}^{\infty} \lambda_{\langle n\rangle}^{\alpha, \beta}\left\langle f, \Phi_{n}^{\alpha, \beta}\right\rangle \Phi_{n}^{\alpha, \beta}
$$

on the domain Dom $\mathbb{L}_{\alpha, \beta}$ consisting of all functions $f \in L^{2}(-\pi, \pi)$ for which the defining series converges in $L^{2}(-\pi, \pi)$; see [25, Section 4].

Next, we gather some facts about potential operators associated with $\mathbb{L}_{\alpha, \beta}$. Let $\sigma>0$. We consider the Riesz type potentials $\mathbb{L}_{\alpha, \beta}^{-\sigma}$ assuming that $\alpha+\beta \neq-1$ (when $\alpha+\beta=-1$, the bottom eigenvalue of $\mathbb{L}_{\alpha, \beta}$ is 0$)$ and the Bessel type potentials ( $\left.\operatorname{Id}+\mathbb{L}_{\alpha, \beta}\right)^{-\sigma}$ with no restrictions 
on $\alpha$ and $\beta$. Clearly, these operators are well defined spectrally and bounded in $L^{2}(-\pi, \pi)$. The spectral decomposition of $\mathbb{L}_{\alpha, \beta}^{-\sigma}$ is given by

$$
\mathbb{L}_{\alpha, \beta}^{-\sigma} f=\sum_{n=0}^{\infty}\left(\lambda_{\langle n\rangle}^{\alpha, \beta}\right)^{-\sigma}\left\langle f, \Phi_{n}^{\alpha, \beta}\right\rangle \Phi_{n}^{\alpha, \beta}, \quad f \in L^{2}(-\pi, \pi) .
$$

Splitting $f$ into its even and odd parts, we can write

$$
\begin{aligned}
\mathbb{L}_{\alpha, \beta}^{-\sigma} f & =\mathbb{L}_{\alpha, \beta}^{-\sigma} f_{\text {even }}+\mathbb{L}_{\alpha, \beta}^{-\sigma} f_{\text {odd }} \\
& =\sum_{n=0}^{\infty}\left(\lambda_{n}^{\alpha, \beta}\right)^{-\sigma}\left\langle f_{\text {even }}, \Phi_{2 n}^{\alpha, \beta}\right\rangle \Phi_{2 n}^{\alpha, \beta}+\sum_{n=0}^{\infty}\left(\lambda_{n+1}^{\alpha, \beta}\right)^{-\sigma}\left\langle f_{\text {odd }}, \Phi_{2 n+1}^{\alpha, \beta}\right\rangle \Phi_{2 n+1}^{\alpha, \beta} \\
& \equiv\left(\mathbb{L}_{\alpha, \beta}^{-\sigma}\right)_{\mathrm{e}} f_{\text {even }}+\left(\mathbb{L}_{\alpha, \beta}^{-\sigma}\right)_{\mathrm{o}} f_{\text {odd }} .
\end{aligned}
$$

This is the decomposition of $\mathbb{L}_{\alpha, \beta}^{-\sigma} f$ into its even and odd parts, since the two terms in (2.3) are even and odd functions, respectively. Clearly, an analogous decomposition holds for $\left(\mathrm{Id}+\mathbb{L}_{\alpha, \beta}\right)^{-\sigma}$. We shall use these facts in the sequel.

Proposition 2.1. Let $\alpha, \beta>-1$ and $\sigma>0$. Assume that $p>p^{\prime}(\alpha, \beta)$ and $1 \leq q<p(\alpha, \beta)$. Then $\mathbb{L}_{\alpha, \beta}^{-\sigma}, \alpha+\beta \neq-1$, and $\left(\operatorname{Id}+\mathbb{L}_{\alpha, \beta}\right)^{-\sigma}$, defined initially on $L^{2}(-\pi, \pi)$, extend to bounded operators from $L^{p}(-\pi, \pi)$ to $L^{q}(-\pi, \pi)$ if and only if

$$
\frac{1}{q} \geq \frac{1}{p}-2 \sigma
$$

Moreover, these operators extend to bounded operators from $L^{p}(-\pi, \pi)$ to $L^{\infty}(-\pi, \pi)$ if and only if

$$
\alpha, \beta \geq-1 / 2 \quad \text { and } \quad \frac{1}{p}<2 \sigma .
$$

Proof. We consider only the Riesz type potentials since the arguments for the Bessel type potentials are parallel. Define the restricted operators acting initially on the smaller space $L^{2}(0, \pi)$ :

$$
\begin{aligned}
& \left(\mathbb{L}_{\alpha, \beta}^{-\sigma}\right)_{\mathrm{e}}^{+} h=\sum_{n=0}^{\infty}\left(\lambda_{n}^{\alpha, \beta}\right)^{-\sigma}\left\langle h,\left(\Phi_{2 n}^{\alpha, \beta}\right)^{+}\right\rangle_{+}\left(\Phi_{2 n}^{\alpha, \beta}\right)^{+}, \quad h \in L^{2}(0, \pi), \\
& \left(\mathbb{L}_{\alpha, \beta}^{-\sigma}\right)_{\mathrm{o}}^{+} h=\sum_{n=0}^{\infty}\left(\lambda_{n+1}^{\alpha, \beta}\right)^{-\sigma}\left\langle h,\left(\Phi_{2 n+1}^{\alpha, \beta}\right)^{+}\right\rangle_{+}\left(\Phi_{2 n+1}^{\alpha, \beta}\right)^{+}, \quad h \in L^{2}(0, \pi) .
\end{aligned}
$$

Since $\|F\|_{q} \simeq\left\|F_{\text {even }}\right\|_{q}+\left\|F_{\text {odd }}\right\|_{q}$, taking into account (2.3) we have, for $f \in L^{2}(-\pi, \pi)$,

$$
\begin{aligned}
\left\|\mathbb{L}_{\alpha, \beta}^{-\sigma} f\right\|_{L^{q}(-\pi, \pi)} & \simeq\left\|\left(\mathbb{L}_{\alpha, \beta}^{-\sigma}\right)_{\mathrm{e}} f_{\text {even }}\right\|_{L^{q}(-\pi, \pi)}+\left\|\left(\mathbb{L}_{\alpha, \beta}^{-\sigma}\right)_{\mathrm{o}} f_{\text {odd }}\right\|_{L^{q}(-\pi, \pi)} \\
& =2^{1 / q}\left(\left\|\left(\mathbb{L}_{\alpha, \beta}^{-\sigma}\right)_{\mathrm{e}}^{+} f_{\text {even }}^{+}\right\|_{L^{q}(0, \pi)}+\left\|\left(\mathbb{L}_{\alpha, \beta}^{-\sigma}\right)_{\mathrm{o}}^{+} f_{\text {odd }}^{+}\right\|_{L^{q}(0, \pi)}\right) .
\end{aligned}
$$

Thus the assertion we must prove is equivalent to the following: $\left(\mathbb{L}_{\alpha, \beta}^{-\sigma}\right)_{\mathrm{e}}^{+}$and $\left(\mathbb{L}_{\alpha, \beta}^{-\sigma}\right)_{\mathrm{o}}^{+}$, defined initially on $L^{2}(0, \pi)$, extend simultaneously to bounded operators from $L^{p}(0, \pi)$ to $L^{q}(0, \pi)$ if and only if $\frac{1}{q} \geq \frac{1}{p}-2 \sigma$; moreover, these operators extend simultaneously to bounded operators from $L^{p}(0, \pi)$ to $L^{\infty}(0, \pi)$ if and only if $\alpha, \beta \geq-1 / 2$ and $\frac{1}{p}<2 \sigma$.

Now it is enough to observe that, in view of (2.2) and the identity $\lambda_{n+1}^{\alpha, \beta}=\lambda_{n}^{\alpha+1, \beta+1}$, the operators $\left(\mathbb{L}_{\alpha, \beta}^{-\sigma}\right)_{\mathrm{e}}^{+}$and $\left(\mathbb{L}_{\alpha, \beta}^{-\sigma}\right)_{\mathrm{o}}^{+}$coincide, up to the constant factor $1 / 2$, with the Riesz type potentials $L_{\alpha, \beta}^{-\sigma}$ and $L_{\alpha+1, \beta+1}^{-\sigma}$ related to $L_{\alpha, \beta}$ and investigated in [19]. The conclusion then follows by [19, Theorem 2.4], see [17, Proposition 2.1]. 
The extensions from Proposition 2.1 are unique provided that $p<\infty$. In this case we denote them by still the same and common symbol $\mathbb{L}_{\alpha, \beta}^{-\sigma}$. It is worth noting that all these extensions are actually realized by an integral operator with a positive kernel. But this fact is irrelevant for our purposes, therefore we omit further details.

Denote

$$
\mathbb{S}_{\alpha, \beta}:=\operatorname{span}\left\{\Phi_{n}^{\alpha, \beta}: n \geq 0\right\} .
$$

Since $\left\{\Phi_{n}^{\alpha, \beta}: n \geq 0\right\}$ is an orthonormal basis, $\mathbb{S}_{\alpha, \beta}$ is dense in $L^{2}(-\pi, \pi)$. The latter property remains true in some $L^{p}$ spaces.

Lemma 2.2. Let $\alpha, \beta>-1$ and $1 \leq p<p(\alpha, \beta)$. Then $\mathbb{S}_{\alpha, \beta}$ is a dense subspace of $L^{p}(-\pi, \pi)$.

Proof. Take $f \in L^{p}(-\pi, \pi)$. It suffices to approximate separately $f_{\text {even }}$ and $f_{\text {odd }}$. Recall that the systems $\left\{\Phi_{2 n}^{\alpha, \beta}: n \geq 0\right\}$ and $\left\{\Phi_{2 n+1}^{\alpha, \beta}: n \geq 0\right\}$ consist of even and odd functions, respectively. Moreover, each of these systems when restricted to $(0, \pi)$ is linearly dense in $L^{p}(0, \pi)$, see $(2.2)$ and [28, Lemma 2.3]. Consequently, one can approximate $f_{\text {even }}$ and $f_{\text {odd }}$ in $L^{p}(-\pi, \pi)$ by finite linear combinations of $\Phi_{2 n}, n \geq 0$, and $\Phi_{2 n+1}, n \geq 0$, respectively.

Lemma 2.3. Let $\alpha, \beta>-1, p \in E(\alpha, \beta)$ and assume that $f \in L^{p}(-\pi, \pi)$. If $\left\langle f, \Phi_{n}^{\alpha, \beta}\right\rangle=0$ for all $n \geq 0$, then $f \equiv 0$.

Proof. It is enough to observe that the lemma holds for $f \in \mathbb{S}_{\alpha, \beta}$ and then use the density of $\mathbb{S}_{\alpha, \beta}$ (see Lemma 2.2) in the dual space $\left(L^{p}(-\pi, \pi)\right)^{*}=L^{p^{\prime}}(-\pi, \pi)$.

Proposition 2.4. Let $\alpha, \beta>-1$ and $p \in E(\alpha, \beta)$. For each $\sigma>0, \mathbb{L}_{\alpha, \beta}^{-\sigma}, \alpha+\beta \neq-1$, and $\left(\mathrm{Id}+\mathbb{L}_{\alpha, \beta}\right)^{-\sigma}$ are injective on $L^{p}(-\pi, \pi)$.

Proof. We focus ourselves on $\mathbb{L}_{\alpha, \beta}^{-\sigma}$ and essentially repeat the arguments from the proof of [4, Proposition 1]. Notice that for $f \in \mathbb{S}_{\alpha, \beta}$

$$
\left\langle\mathbb{L}_{\alpha, \beta}^{-\sigma} f, \Phi_{n}^{\alpha, \beta}\right\rangle=\left(\lambda_{\langle n\rangle}^{\alpha, \beta}\right)^{-\sigma}\left\langle f, \Phi_{n}^{\alpha, \beta}\right\rangle, \quad n \geq 0
$$

By Hölder's inequality and the $L^{p}$-boundedness of $\mathbb{L}_{\alpha, \beta}^{-\sigma}$ (see Proposition 2.1), the functionals

$$
f \mapsto\left\langle\mathbb{L}_{\alpha, \beta}^{-\sigma} f, \Phi_{n}^{\alpha, \beta}\right\rangle \quad \text { and } \quad f \mapsto\left\langle f, \Phi_{n}^{\alpha, \beta}\right\rangle
$$

are bounded from $L^{p}(-\pi, \pi)$ to $\mathbb{C}$. Since $\mathbb{S}_{\alpha, \beta}$ is dense in $L^{p}(-\pi, \pi)$, we infer that (2.4) holds for $f \in L^{p}(-\pi, \pi)$. Now, if $\mathbb{L}_{\alpha, \beta}^{-\sigma} f \equiv 0$ for some $f \in L^{p}(-\pi, \pi)$, then (2.4) implies $\left\langle f, \Phi_{n}^{\alpha, \beta}\right\rangle=0$ for all $n \geq 0$ and hence Lemma 2.3 gives $f \equiv 0$. Thus $\mathbb{L}_{\alpha, \beta}^{-\sigma}$ is one-to-one on $L^{p}(-\pi, \pi)$.

Now we can define the Jacobi potential spaces as the ranges of the potential operators on $L^{p}(-\pi, \pi)$,

$$
\mathcal{L}_{\alpha, \beta}^{p, s}(-\pi, \pi):= \begin{cases}\mathbb{L}_{\alpha, \beta}^{-s / 2}\left(L^{p}(-\pi, \pi)\right), & \alpha+\beta \neq-1, \\ \left(\operatorname{Id}+\mathbb{L}_{\alpha, \beta}\right)^{-s / 2}\left(L^{p}(-\pi, \pi)\right), & \alpha+\beta=-1,\end{cases}
$$

where $p \in E(\alpha, \beta)$ and $s>0$. Then the formula

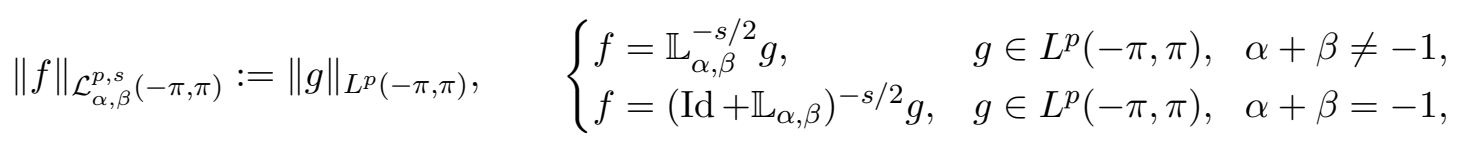

defines a norm on $\mathcal{L}_{\alpha, \beta}^{p, s}(-\pi, \pi)$ and it is straightforward to check that $\mathcal{L}_{\alpha, \beta}^{p, s}(-\pi, \pi)$ equipped with this norm is a Banach space. 
In order to give a suitable definition of Sobolev spaces in the symmetrized setting we need to understand the structure of the potential spaces. The following result describes the symmetrized potential spaces in terms of the potential spaces related to the initial, non-symmetrized situation. The latter spaces are defined similarly as $\mathcal{L}_{\alpha, \beta}^{p, s}(-\pi, \pi)$, see [16, 17] for details.

Proposition 2.5. Let $\alpha, \beta>-1, p \in E(\alpha, \beta)$ and $s>0$. Then $f \in \mathcal{L}_{\alpha, \beta}^{p, s}(-\pi, \pi)$ if and only if $f_{\text {even }}^{+} \in \mathcal{L}_{\alpha, \beta}^{p, s}(0, \pi)$ and $f_{\text {odd }}^{+} \in \mathcal{L}_{\alpha+1, \beta+1}^{p, s}(0, \pi)$. Moreover,

$$
\|f\|_{\mathcal{L}_{\alpha, \beta}^{p, s}(-\pi, \pi)} \simeq\left\|f_{\text {even }}^{+}\right\|_{\mathcal{L}_{\alpha, \beta}^{p, s}(0, \pi)}+\left\|f_{\text {odd }}^{+}\right\|_{\mathcal{L}_{\alpha+1, \beta+1}^{p, s}(0, \pi)} .
$$

Proof. We assume that $\alpha+\beta \neq-1$, the opposite case requires only minor modifications which are left to the reader. Let $f \in \mathcal{L}_{\alpha, \beta}^{p, s}(-\pi, \pi)$. This means that there is $g \in L^{p}(-\pi, \pi)$ such that $f=\mathbb{L}_{\alpha, \beta}^{-s / 2} g$; then $\|f\|_{\mathcal{L}_{\alpha, \beta}^{p, s}(-\pi, \pi)}=\|g\|_{L^{p}(-\pi, \pi)}$.

Assume, for the time being, that $g$ belongs also to $L^{2}(-\pi, \pi)$. Then from $(2.3)$ we see that

$$
f_{\text {even }}=\left(\mathbb{L}_{\alpha, \beta}^{-s / 2}\right)_{\mathrm{e}} g_{\text {even }}, \quad f_{\text {odd }}=\left(\mathbb{L}_{\alpha, \beta}^{-s / 2}\right)_{\mathrm{o}} g_{\text {odd }} .
$$

Thus

$$
\begin{aligned}
f_{\text {even }}^{+} & =\sum_{n=0}^{\infty}\left(\lambda_{n}^{\alpha, \beta}\right)^{-s / 2}\left\langle g_{\text {even }}, \Phi_{2 n}^{\alpha, \beta}\right\rangle\left(\Phi_{2 n}^{\alpha, \beta}\right)^{+}=2 \sum_{n=0}^{\infty}\left(\lambda_{n}^{\alpha, \beta}\right)^{-s / 2}\left\langle g_{\text {even }}^{+},\left(\Phi_{2 n}^{\alpha, \beta}\right)^{+}\right\rangle_{+}\left(\Phi_{2 n}^{\alpha, \beta}\right)^{+} \\
& =L_{\alpha, \beta}^{-s / 2} g_{\text {even }}^{+}
\end{aligned}
$$

where the last identity is a consequence of (2.2). Similarly, $f_{\text {odd }}^{+}=L_{\alpha+1, \beta+1}^{-s / 2} g_{\text {odd }}^{+}$.

A general $g \in L^{p}(-\pi, \pi)$ can be approximated in the $L^{p}$ norm by functions from $L^{p} \cap$ $L^{2}(-\pi, \pi)$. Then combining the above with the $L^{p}(-\pi, \pi)$-boundedness of $\mathbb{L}_{\alpha, \beta}^{-s / 2}$ and $L^{p}(0, \pi)$ boundedness of $L_{\alpha, \beta}^{-s / 2}$ and $L_{\alpha+1, \beta+1}^{-s / 2}$, we get

$$
f_{\text {even }}^{+}=L_{\alpha, \beta}^{-s / 2} g_{\text {even }}^{+}, \quad f_{\text {odd }}^{+}=L_{\alpha+1, \beta+1}^{-s / 2} g_{\text {odd }}^{+},
$$

in the general case. Since

$$
\|g\|_{L^{p}(-\pi, \pi)} \simeq\left\|g_{\mathrm{even}}^{+}\right\|_{L^{p}(0, \pi)}+\left\|g_{\text {odd }}^{+}\right\|_{L^{p}(0, \pi)}, \quad g \in L^{p}(-\pi, \pi),
$$

we see that $f_{\text {even }}^{+} \in \mathcal{L}_{\alpha, \beta}^{p, s}(0, \pi), f_{\text {odd }}^{+} \in \mathcal{L}_{\alpha+1, \beta+1}^{p, s}(0, \pi)$ and, moreover, (2.5) holds.

The opposite implication is verified along similar lines. Given a function $f$ on $(-\pi, \pi)$, assume that $f_{\text {even }}^{+} \in \mathcal{L}_{\alpha, \beta}^{p, s}(0, \pi)$ and $f_{\text {odd }}^{+} \in \mathcal{L}_{\alpha+1, \beta+1}^{p, s}(0, \pi)$. Then $f_{\text {even }}^{+}=L_{\alpha, \beta}^{-s / 2} h$ and $f_{\text {odd }}^{+}=L_{\alpha+1, \beta+1}^{-s / 2} \widetilde{h}$ for some $h, \widetilde{h} \in L^{p}(0, \pi)$. Extending $h$ and $\widetilde{h}$ to even and odd functions on $(-\pi, \pi)$, respectively, we let $g$ be the sum of these extensions. Then we find that $f_{\text {even }}^{+}=\left(\mathbb{L}_{\alpha, \beta}^{-s / 2} g\right)_{\text {even }}^{+}, f_{\text {odd }}^{+}=$ $\left(\mathbb{L}_{\alpha, \beta}^{-s / 2} g\right)_{\text {odd }}^{+}$, and consequently $f=\mathbb{L}_{\alpha, \beta}^{-s / 2} g$ with $g \in L^{p}(-\pi, \pi)$. Thus $f \in \mathcal{L}_{\alpha, \beta}^{p, s}(-\pi, \pi)$.

\section{Sobolev spaces}

Our aim in this section is to establish a suitable definition of Sobolev spaces in the symmetrized setting. Here "suitable" means existence of an isomorphism between the Sobolev spaces and the potential spaces with properly chosen parameters. Note that such an isomorphism gives also a characterization of the potential spaces with some parameters in terms of appropriate higher-order 'derivatives'. 
According to a general concept, Sobolev spaces $\mathbb{W}_{\alpha, \beta}^{p, m}, m \geq 1$ integer, associated with $\mathbb{L}_{\alpha, \beta}$, should be defined by

$$
\mathbb{W}_{\alpha, \beta}^{p, m}:=\left\{f \in L^{p}(-\pi, \pi): \mathfrak{D}_{\alpha, \beta}^{(k)} f \in L^{p}(-\pi, \pi), k=1, \ldots, m\right\}
$$

and equipped with the norm

$$
\|f\|_{\mathbb{W}_{\alpha, \beta}^{p, m}}:=\sum_{k=0}^{m}\left\|\mathfrak{D}_{\alpha, \beta}^{(k)} f\right\|_{L^{p}(-\pi, \pi)} .
$$

Here $\mathfrak{D}_{\alpha, \beta}^{(k)}$ is a suitably defined differential-difference operator of order $k$ playing the role of higher-order derivative, with the differentiation understood in a weak sense; we use the convention $\mathfrak{D}_{\alpha, \beta}^{(0)}:=\mathrm{Id}$. So the question is how to choose $\mathfrak{D}_{\alpha, \beta}^{(k)}$.

It turns out that the seemingly most natural choice $\mathfrak{D}_{\alpha, \beta}^{(k)}=\mathbb{D}_{\alpha, \beta}^{k}$ is not appropriate. Another quite natural attempt is to mimic the variable index derivatives, which lead to a good definition of Sobolev spaces in the non-symmetrized setting, see [16, Section 2]. Unfortunately, taking $\mathfrak{D}_{\alpha, \beta}^{(k)}=\mathbb{D}_{\alpha+k-1, \beta+k-1} \circ \cdots \circ \mathbb{D}_{\alpha+1, \beta+1} \circ \mathbb{D}_{\alpha, \beta}$ is inappropriate as well. Counterexamples for these choices are discussed at the end of this section.

To find suitable higher-order 'derivatives' in the symmetrized setting we first introduce the variable index higher-order 'derivatives'

$$
\mathfrak{d}_{\alpha, \beta}^{(k)}:=D_{\alpha+k-1, \beta+k-1} \circ \cdots \circ D_{\alpha+1, \beta+1} \circ D_{\alpha, \beta}, \quad k \geq 1,
$$

acting on functions on $(-\pi, \pi)$ or $(0, \pi)$; we set $\mathfrak{d}_{\alpha, \beta}^{(0)}:=$ Id. In [16, Theorem A] we proved that in the non-symmetrized Jacobi function setting the Sobolev spaces

$$
W_{\alpha, \beta}^{p, m}(0, \pi)=\left\{h \in L^{p}(0, \pi): \mathfrak{d}_{\alpha, \beta}^{(k)} h \in L^{p}(0, \pi), k=1, \ldots, m\right\}
$$

equipped with the norm

$$
\|h\|_{W_{\alpha, \beta}^{p, m}(0, \pi)}=\sum_{k=0}^{m}\left\|\mathfrak{d}_{\alpha, \beta}^{(k)} h\right\|_{L^{p}(0, \pi)},
$$

are isomorphic to the potential spaces $\mathcal{L}_{\alpha, \beta}^{p, m}(0, \pi)$. Combining this result with Proposition 2.5 we get the following.

Proposition 3.1. Let $\alpha, \beta>-1, p \in E(\alpha, \beta)$ and $m \geq 1$ be integer. Then $f \in \mathcal{L}_{\alpha, \beta}^{p, m}(-\pi, \pi)$ if and only if $f_{\mathrm{even}}^{+} \in W_{\alpha, \beta}^{p, m}(0, \pi)$ and $f_{\mathrm{odd}}^{+} \in W_{\alpha+1, \beta+1}^{p, m}(0, \pi)$. Moreover,

$$
\|f\|_{\mathcal{L}_{\alpha, \beta}^{p, m}(-\pi, \pi)} \simeq\left\|f_{\text {even }}^{+}\right\|_{W_{\alpha, \beta}^{p, m}(0, \pi)}+\left\|f_{\text {odd }}^{+}\right\|_{W_{\alpha+1, \beta+1}^{p, m}(0, \pi)} .
$$

This motivates the following definition of the higher-order 'derivatives' $\mathfrak{D}_{\alpha, \beta}^{(k)}$.

Definition 3.2. For $\mathrm{k}=0,1,2, \ldots$, let

$$
\mathfrak{D}_{\alpha, \beta}^{(k)} f:=\mathfrak{d}_{\alpha, \beta}^{(k)} f_{\text {even }}+\mathfrak{d}_{\alpha+1, \beta+1}^{(k)} f_{\text {odd }}
$$

Note that the 'derivatives' $\mathfrak{D}_{\alpha, \beta}^{(k)}$ are counterintuitive from the point of view of the symmetrization concept, since they do not express via compositions of the symmetrized 'derivative' $\mathbb{D}_{\alpha, \beta}$. Nevertheless, we have the following result. 
Theorem 3.3. Let $\alpha, \beta>-1, p \in E(\alpha, \beta)$ and $m \geq 1$ be integer. Then

$$
\mathbb{W}_{\alpha, \beta}^{p, m}=\mathcal{L}_{\alpha, \beta}^{p, m}(-\pi, \pi)
$$

in the sense of isomorphism of Banach spaces.

Proof. Let $k \geq 0$. For symmetry reasons, we have $\mathfrak{D}_{\alpha, \beta}^{(k)} f \in L^{p}(-\pi, \pi)$ if and only if $\mathfrak{d}_{\alpha, \beta}^{(k)} f_{\text {even }}^{+} \in$ $L^{p}(0, \pi)$ and $\mathfrak{d}_{\alpha+1, \beta+1}^{(k)} f_{\text {odd }}^{+} \in L^{p}(0, \pi)$. Furthermore,

$$
\left\|\mathfrak{D}_{\alpha, \beta}^{(k)} f\right\|_{L^{p}(-\pi, \pi)} \simeq\left\|\mathfrak{d}_{\alpha, \beta}^{(k)} f_{\text {even }}^{+}\right\|_{L^{p}(0, \pi)}+\left\|\mathfrak{d}_{\alpha+1, \beta+1}^{(k)} f_{\text {odd }}^{+}\right\|_{L^{p}(0, \pi)} .
$$

Thus the assertion follows from Proposition 3.1.

In the remaining part of this section we look closer at the two already mentioned, seemingly more natural concepts of Sobolev spaces in the symmetrized setting, which in general fail to be isomorphic with the corresponding potential spaces. For $m \geq 1$ denote

$$
\begin{aligned}
& \mathcal{W}_{\alpha, \beta}^{p, m}:=\left\{f \in L^{p}(-\pi, \pi): \mathbb{D}_{\alpha, \beta}^{k} f \in L^{p}(-\pi, \pi), k=1, \ldots, m\right\}, \\
& \mathfrak{W}_{\alpha, \beta}^{p, m}:=\left\{f \in L^{p}(-\pi, \pi): \mathbb{D}_{\alpha+k-1, \beta+k-1} \cdots \mathbb{D}_{\alpha+1, \beta+1} \mathbb{D}_{\alpha, \beta} f \in L^{p}(-\pi, \pi), k=1, \ldots, m\right\},
\end{aligned}
$$

and equip these spaces with the natural norms.

Proposition 3.4. Let $\alpha, \beta>-1$ and $p \in E(\alpha, \beta)$. For every $\alpha, \beta<1 / p-1 / 2$ there exists $f \in \mathcal{W}_{\alpha, \beta}^{p, 1}=\mathfrak{W}_{\alpha, \beta}^{p, 1}$ such that $f \notin \mathcal{L}_{\alpha, \beta}^{p, 1}(-\pi, \pi)$. Furthermore, if $\alpha \leq-1 / p+1 / 2$ or $\beta \leq-1 / p+1 / 2$ then there exists $g \in \mathcal{L}_{\alpha, \beta}^{p, 2}(-\pi, \pi)$ such that $g \notin \mathfrak{W}_{\alpha, \beta}^{p, 2}$.

Proof. Let

$$
f(\theta)=\operatorname{sign}(\theta) \Psi^{-\alpha-1,-\beta-1}(\theta)=\operatorname{sign}(\theta)\left|\sin \frac{\theta}{2}\right|^{-\alpha-1 / 2}\left(\cos \frac{\theta}{2}\right)^{-\beta-1 / 2} .
$$

Since $f$ is odd, we have $\left(\mathbb{D}_{\alpha, \beta} f\right)^{+}=D_{\alpha, \beta}^{*} f^{+}$and $\left[16\right.$, formula (9)] reveals that $D_{\alpha, \beta}^{*} f^{+}$vanishes. Therefore $f^{+}, D_{\alpha, \beta}^{*} f^{+} \in L^{p}(0, \pi)$ for $\alpha, \beta$ in question. By symmetry it follows that $f \in \mathcal{W}_{\alpha, \beta}^{p, 1}$.

On the other hand, with the aid of [16, formula (8)] we find that

$$
\left(\mathfrak{D}_{\alpha, \beta}^{(1)} f\right)^{+}(\theta)=\left(\mathfrak{d}_{\alpha+1, \beta+1}^{(1)} f\right)^{+}(\theta) \gtrsim \theta^{-\alpha-3 / 2}(\pi-\theta)^{-\beta-3 / 2}, \quad \theta \in(0, \pi) .
$$

Thus $\left(\mathfrak{D}_{\alpha, \beta}^{(1)} f\right)^{+} \notin L^{p}(0, \pi)$, in view of the assumption $p \in E(\alpha, \beta)$. This implies $f \notin \mathbb{W}_{\alpha, \beta}^{p, 1}$. By Theorem 3.3, $f \notin \mathcal{L}_{\alpha, \beta}^{p, 1}(-\pi, \pi)$.

To prove the second claim let

$$
g(\theta)=\operatorname{sign}(\theta) \Psi^{\alpha+1, \beta+1}(\theta)=\operatorname{sign}(\theta)\left|\sin \frac{\theta}{2}\right|^{\alpha+3 / 2}\left(\cos \frac{\theta}{2}\right)^{\beta+3 / 2} .
$$

Since $g$ is odd, we have $\left(\mathbb{D}_{\alpha+1, \beta+1} \mathbb{D}_{\alpha, \beta} g\right)^{+}=D_{\alpha+1, \beta+1} D_{\alpha, \beta}^{*} g^{+}$. Using [16, formulas (8) and (9)] we get

$$
\left(\mathbb{D}_{\alpha+1, \beta+1} \mathbb{D}_{\alpha, \beta} g\right)^{+}(\theta) \gtrsim \theta^{\alpha-1 / 2}(\pi-\theta)^{\beta-1 / 2}, \quad \theta \in(0, \pi) .
$$

Consequently, for the assumed range of $\alpha, \beta$ we have that $\left(\mathbb{D}_{\alpha+1, \beta+1} \mathbb{D}_{\alpha, \beta} g\right)^{+}$is not in $L^{p}(0, \pi)$. Therefore, by symmetry, $g \notin \mathfrak{W}_{\alpha, \beta}^{p, 2}$.

On the other hand $\left(\mathfrak{D}_{\alpha, \beta}^{(1)} g\right)^{+}=\left(\mathfrak{d}_{\alpha+1, \beta+1}^{(1)} g\right)^{+}=D_{\alpha+1, \beta+1} g^{+}=0$, by [16, formula (8)]. Consequently, $\left(\mathfrak{D}_{\alpha, \beta}^{(2)} g\right)^{+}=D_{\alpha+2, \beta+2} D_{\alpha+1, \beta+1} g^{+}=0$. Since $g^{+} \in L^{p}(0, \pi)$, using again the symmetry we see that $g \in \mathbb{W}_{\alpha, \beta}^{p, 2}$. This together with Theorem 3.3 implies $g \in \mathcal{L}_{\alpha, \beta}^{p, 2}(-\pi, \pi)$. 
Although Proposition 3.4 shows that the spaces $\mathcal{W}_{\alpha, \beta}^{p, m}$ and $\mathcal{L}_{\alpha, \beta}^{p, m}(-\pi, \pi)$ do not coincide in general, one might still wonder what the relation between them is, if any. The answer is given by the next result.

Proposition 3.5. Let $\alpha, \beta>-1, p \in E(\alpha, \beta)$ and $m \geq 1$ be integer. Then

$$
\mathcal{L}_{\alpha, \beta}^{p, m}(-\pi, \pi) \subset \mathcal{W}_{\alpha, \beta}^{p, m}
$$

in the sense of embedding of Banach spaces.

The proof of Proposition 3.5 involves higher-order Riesz transforms of the following form. For $k \geq 1$ integer, let

$$
\mathbb{R}_{\alpha, \beta}^{k}= \begin{cases}\mathbb{D}_{\alpha, \beta}^{k} \mathbb{L}_{\alpha, \beta}^{-k / 2}, & \alpha+\beta \neq-1, \\ \mathbb{D}_{\alpha, \beta}^{k}\left(\mathrm{Id}+\mathbb{L}_{\alpha, \beta}\right)^{-k / 2}, & \alpha+\beta=-1 .\end{cases}
$$

Clearly, $\mathbb{R}_{\alpha, \beta}^{k}$ is well defined on $\mathbb{S}_{\alpha, \beta}$. But we also need to know that each $\mathbb{R}_{\alpha, \beta}^{k}, k \geq 1$, extends to a bounded operator on $L^{p}(-\pi, \pi)$.

Lemma 3.6. Let $\alpha, \beta>-1, p \in E(\alpha, \beta)$ and $k \geq 1$. Then the operator

$$
f \mapsto \mathbb{R}_{\alpha, \beta}^{k} f, \quad f \in \mathbb{S}_{\alpha, \beta},
$$

extends uniquely to a bounded linear operator on $L^{p}(-\pi, \pi)$.

Assuming that this result holds, we now give a short proof of Proposition 3.5. Lemma 3.6 will be shown subsequently.

Proof of Proposition 3.5. We may assume that $\alpha+\beta \neq-1$, since treatment of the opposite case is analogous. Let $f \in \mathcal{L}_{\alpha, \beta}^{p, m}(-\pi, \pi)$. Then $f=\mathbb{L}_{\alpha, \beta}^{-m / 2} g$ for some $g \in L^{p}(-\pi, \pi)$. By the $L^{p}$-boundedness of $\mathbb{L}_{\alpha, \beta}^{-(m-k) / 2}$ (see Proposition 2.1) and Lemma 3.6, for any $0 \leq k \leq m$ we have

$$
\begin{aligned}
\left\|\mathbb{D}_{\alpha, \beta}^{k} f\right\|_{L^{p}(-\pi, \pi)} & =\left\|\mathbb{D}_{\alpha, \beta}^{k} \mathbb{L}_{\alpha, \beta}^{-m / 2} g\right\|_{L^{p}(-\pi, \pi)}=\left\|\mathbb{R}_{\alpha, \beta}^{k} \mathbb{L}_{\alpha, \beta}^{-(m-k) / 2} g\right\|_{L^{p}(-\pi, \pi)} \\
& \lesssim\|g\|_{L^{p}(-\pi, \pi)}=\|f\|_{\mathcal{L}_{\alpha, \beta}^{p, m}(-\pi, \pi)}
\end{aligned}
$$

where $\mathbb{R}_{\alpha, \beta}^{k}$ stands for the extension provided by Lemma 3.6 (with the natural interpretation $\mathbb{R}_{\alpha, \beta}^{0}=\mathrm{Id}$ ). The second identity above is easily justified when $g \in \mathbb{S}_{\alpha, \beta}$, and then it carries over to general $g$ by continuity. The conclusion follows.

It remains to prove Lemma 3.6. The argument relies on a multiplier-transplantation theorem due to Muckenhoupt [18], see [16, Lemma 2.1]. Here we merely sketch the proof, leaving the details to interested readers.

Proof of Lemma 3.6. Assume that $\alpha+\beta \neq-1$ (the opposite case is similar) and take $f \in \mathbb{S}_{\alpha, \beta}$. We have

$$
\mathbb{R}_{\alpha, \beta}^{k} f=-(-1)^{\langle k\rangle} \underbrace{\cdots D_{\alpha, \beta}^{*} D_{\alpha, \beta}}_{k \text { components }}\left(\mathbb{L}_{\alpha, \beta}^{-k / 2} f\right)_{\text {even }}+(-1)^{\langle k\rangle} \underbrace{\cdots D_{\alpha, \beta} D_{\alpha, \beta}^{*}}_{k \text { components }}\left(\mathbb{L}_{\alpha, \beta}^{-k / 2} f\right)_{\text {odd }} .
$$

This is the decomposition of $\mathbb{R}_{\alpha, \beta}^{k} f$ into its even and odd parts, respectively, or vice versa, depending on whether $k$ is even or odd. Since (see the proof of Proposition 2.5)

$$
\left(\mathbb{L}_{\alpha, \beta}^{-k / 2} f\right)_{\text {even }}^{+}=L_{\alpha, \beta}^{-k / 2} f_{\text {even }}^{+}, \quad\left(\mathbb{L}_{\alpha, \beta}^{-k / 2} f\right)_{\text {odd }}^{+}=L_{\alpha+1, \beta+1}^{-k / 2} f_{\text {odd }}^{+},
$$


it suffices to show the bounds

$$
\begin{aligned}
& \|\underbrace{\cdots D_{\alpha, \beta}^{*} D_{\alpha, \beta}}_{k \text { components }} L_{\alpha, \beta}^{-k / 2} h\|_{L^{p}(0, \pi)} \lesssim\|h\|_{L^{p}(0, \pi)}, \quad h \in \operatorname{span}\left\{\phi_{n}^{\alpha, \beta}: n \geq 0\right\}, \\
& \|\underbrace{\cdots D_{\alpha, \beta} D_{\alpha, \beta}^{*}}_{k \text { components }} L_{\alpha+1, \beta+1}^{-k / 2} h\|_{L^{p}(0, \pi)} \lesssim\|h\|_{L^{p}(0, \pi)}, \quad h \in \operatorname{span}\left\{\phi_{n}^{\alpha+1, \beta+1}: n \geq 0\right\} .
\end{aligned}
$$

Here (3.1) is contained in [16, Proposition 4.2], since the underlying operator coincides with the Riesz transform $\mathcal{R}_{\alpha, \beta}^{k}$ considered in [16]. So it remains to verify (3.2).

Taking into account $[16$, formulas (5) and (6)] one finds that

$$
\underbrace{\cdots D_{\alpha, \beta} D_{\alpha, \beta}^{*}}_{k \text { components }} L_{\alpha+1, \beta+1}^{-k / 2} h=\sum_{n=0}^{\infty}\left(1-\frac{A_{\alpha, \beta}^{2}}{\lambda_{n+1}^{\alpha, \beta}}\right)^{-k / 2}\left\langle h, \phi_{n}^{\alpha+1, \beta+1}\right\rangle_{+} \begin{cases}\phi_{n}^{\alpha+1, \beta+1}, & k \text { even, } \\ -\phi_{n+1}^{\alpha, \beta}, & k \text { odd. }\end{cases}
$$

Now (3.2) follows from a special case of Muckenhoupt's multiplier-transplantation theorem [16, Lemma 2.1]; see, e.g., the proof of [16, Proposition 3.4].

\section{Characterization of potential spaces via fractional square functions}

In this section we give necessary and sufficient conditions, expressed in terms of suitably defined fractional square functions, for a function to belong to the potential space $\mathcal{L}_{\alpha, \beta}^{p, s}(-\pi, \pi)$. For the sake of brevity, we restrict our main attention to the case $\alpha+\beta \neq-1$. Nevertheless, after a slight modification the result is valid also when $\alpha+\beta=-1$. This issue is discussed at the end of this section.

Let $\left\{\mathbb{H}_{t}^{\alpha, \beta}: t \geq 0\right\}$ be the symmetrized Poisson-Jacobi semigroup, i.e., the semigroup of operators generated by $-\mathbb{L}_{\alpha, \beta}^{1 / 2}$. In view of the spectral theorem, for $f \in L^{2}(-\pi, \pi)$ and $t \geq 0$ we have

$$
\mathbb{H}_{t}^{\alpha, \beta} f=\sum_{n=0}^{\infty} \exp \left(-t \sqrt{\lambda_{\langle n\rangle}^{\alpha, \beta}}\right)\left\langle f, \Phi_{n}^{\alpha, \beta}\right\rangle \Phi_{n}^{\alpha, \beta},
$$

the convergence being in $L^{2}(-\pi, \pi)$. By means of (2.2) and [16, Estimate (1)] one verifies that for $t>0$ the above series converges in fact pointwise in $(-\pi, \pi) \backslash\{0\}$ and, moreover, may serve as a pointwise definition of $\mathbb{H}_{t}^{\alpha, \beta} f$ on $(-\pi, \pi) \backslash\{0\}, t>0$, for $f \in L^{p}(-\pi, \pi), p>p^{\prime}(\alpha, \beta)$. In the latter case, the resulting function $\mathbb{H}_{t}^{\alpha, \beta} f(\theta)$ is smooth in $(t, \theta) \in(0, \infty) \times[(-\pi, \pi) \backslash\{0\}]$. There is also an integral representation of $\mathbb{H}_{t}^{\alpha, \beta} f, t>0$, for $f$ as above, but it will not be needed for our purposes.

Following Segovia and Wheeden [27] and Betancor et al. [5], see also [17], we consider the fractional square function

$$
\mathfrak{g}_{\alpha, \beta}^{\gamma, k} f(\theta)=\left(\int_{0}^{\infty}\left|t^{k-\gamma} \frac{\partial^{k}}{\partial t^{k}} \mathbb{H}_{t}^{\alpha, \beta} f(\theta)\right|^{2} \frac{d t}{t}\right)^{1 / 2}, \quad \theta \in(-\pi, \pi) \backslash\{0\},
$$

where $0<\gamma<k$ and $k=1,2, \ldots$. Notice that $\mathfrak{g}_{\alpha, \beta}^{\gamma, k} f$ is well defined pointwise whenever $f \in L^{p}(-\pi, \pi)$ and $p>p^{\prime}(\alpha, \beta)$. An analogue of $\mathfrak{g}_{\alpha, \beta}^{\gamma, k}$ was investigated in the non-symmetrized Jacobi function setting in [17] and denoted by $g_{\alpha, \beta}^{\gamma, k}$ there. Recall that

$$
g_{\alpha, \beta}^{\gamma, k} h(\theta)=\left(\int_{0}^{\infty}\left|t^{k-\gamma} \frac{\partial^{k}}{\partial t^{k}} H_{t}^{\alpha, \beta} h(\theta)\right|^{2} \frac{d t}{t}\right)^{1 / 2}, \quad \theta \in(0, \pi),
$$


where $\gamma$ and $k$ are as before, and $\left\{H_{t}^{\alpha, \beta}\right\}$ is the Poisson-Jacobi semigroup. See [17] for more details on $H_{t}^{\alpha, \beta}$ and $g_{\alpha, \beta}^{\gamma, k}$.

A simple combination of Proposition 2.5 and [17, Theorem 4.1] allows us to get the following description of the symmetrized potential spaces $\mathcal{L}_{\alpha, \beta}^{p, s}(-\pi, \pi)$ in terms of the non-symmetrized square functions $g_{\alpha, \beta}^{\gamma, k}$.

Proposition 4.1. Let $\alpha, \beta>-1$ be such that $\alpha+\beta \neq-1$ and let $p \in E(\alpha, \beta)$. Fix $0<\gamma<k$ with $k \in \mathbb{N}$. Then $f \in \mathcal{L}_{\alpha, \beta}^{p, \gamma}(-\pi, \pi)$ if and only if $f \in L^{p}(-\pi, \pi)$ and $g_{\alpha, \beta}^{\gamma, k} f_{\text {even }}^{+}, g_{\alpha+1, \beta+1}^{\gamma, k} f_{\text {odd }}^{+} \in L^{p}(0, \pi)$. Moreover,

$$
\|f\|_{\mathcal{L}_{\alpha, \beta}^{p, \gamma}(-\pi, \pi)} \simeq\left\|g_{\alpha, \beta}^{\gamma, k} f_{\text {even }}^{+}\right\|_{L^{p}(0, \pi)}+\left\|g_{\alpha+1, \beta+1}^{\gamma, k} f_{\text {odd }}^{+}\right\|_{L^{p}(0, \pi)}, \quad f \in \mathcal{L}_{\alpha, \beta}^{p, \gamma}(-\pi, \pi) .
$$

This leads to the following characterization of the symmetrized potential spaces.

Theorem 4.2. Let $\alpha, \beta>-1$ be such that $\alpha+\beta \neq-1$ and let $p \in E(\alpha, \beta)$. Fix $0<\gamma<k$ with $k \in \mathbb{N}$. Then $f \in \mathcal{L}_{\alpha, \beta}^{p, \gamma}(-\pi, \pi)$ if and only if $f \in L^{p}(-\pi, \pi)$ and $\mathfrak{g}_{\alpha, \beta}^{\gamma, k} f \in L^{p}(-\pi, \pi)$. Moreover,

$$
\|f\|_{\mathcal{L}_{\alpha, \beta}^{p, \gamma}(-\pi, \pi)} \simeq\left\|\mathfrak{g}_{\alpha, \beta}^{\gamma, k} f\right\|_{L^{p}(-\pi, \pi)}, \quad f \in \mathcal{L}_{\alpha, \beta}^{p, \gamma}(-\pi, \pi) .
$$

Proof. Taking into account Proposition 4.1, it suffices to show the following. Given $p \in$ $E(\alpha, \beta)$,

$$
\left\|\mathfrak{g}_{\alpha, \beta}^{\gamma, k} f\right\|_{L^{p}(-\pi, \pi)} \simeq\left\|g_{\alpha, \beta}^{\gamma, k} f_{\text {even }}^{+}\right\|_{L^{p}(0, \pi)}+\left\|g_{\alpha+1, \beta+1}^{\gamma, k} f_{\text {odd }}^{+}\right\|_{L^{p}(0, \pi)},
$$

uniformly in $f \in L^{p}(-\pi, \pi)$, possibly with infinite values on both sides for some $f$.

To proceed, observe that

$$
\begin{aligned}
& \left(\mathbb{H}_{t}^{\alpha, \beta} f_{\text {even }}\right)^{+}(\theta)=\left(\mathbb{H}_{t}^{\alpha, \beta} f\right)_{\text {even }}^{+}(\theta)=H_{t}^{\alpha, \beta} f_{\text {even }}^{+}(\theta), \quad \theta \in(0, \pi), \\
& \left(\mathbb{H}_{t}^{\alpha, \beta} f_{\text {odd }}\right)^{+}(\theta)=\left(\mathbb{H}_{t}^{\alpha, \beta} f\right)_{\text {odd }}^{+}(\theta)=H_{t}^{\alpha+1, \beta+1} f_{\text {odd }}^{+}(\theta), \quad \theta \in(0, \pi) .
\end{aligned}
$$

These identities are easily verified by means of the series representations of $\mathbb{H}_{t}^{\alpha, \beta}$ and $H_{t}^{\alpha, \beta}$, since the relevant series converge pointwise.

Next, we claim that

$$
\mathfrak{g}_{\alpha, \beta}^{\gamma, k} f(\theta) \leq \mathfrak{g}_{\alpha, \beta}^{\gamma, k} f_{\text {even }}(\theta)+\mathfrak{g}_{\alpha, \beta}^{\gamma, k} f_{\text {odd }}(\theta) \leq \mathfrak{g}_{\alpha, \beta}^{\gamma, k} f(\theta)+\mathfrak{g}_{\alpha, \beta}^{\gamma, k} f(-\theta)
$$

Here the lower bound is clear, since $\mathfrak{g}_{\alpha, \beta}^{\gamma, k}$ is sublinear. To see the upper bound, we first observe that the operators $\mathbb{H}_{t}^{\alpha, \beta}, t>0$, commute with reflections. It is enough to verify this on $\mathbb{S}_{\alpha, \beta}$. Since $\breve{\Phi}_{n}^{\alpha, \beta}=\Phi_{n}^{\alpha, \beta}$ for $n$ even and $\check{\Phi}_{n}^{\alpha, \beta}=-\Phi_{n}^{\alpha, \beta}$ for $n$ odd, we can write

$$
\mathbb{H}_{t}^{\alpha, \beta} \check{\Phi}_{n}^{\alpha, \beta}(\theta)=\exp \left(-t \sqrt{\lambda_{\langle n\rangle}^{\alpha, \beta}}\right)(-1)^{n} \Phi_{n}^{\alpha, \beta}(\theta)=\exp \left(-t \sqrt{\lambda_{\langle n\rangle}^{\alpha, \beta}}\right) \Phi_{n}^{\alpha, \beta}(-\theta)=\mathbb{H}_{t}^{\alpha, \beta} \Phi_{n}^{\alpha, \beta}(-\theta) .
$$

Consequently, $\mathfrak{g}_{\alpha, \beta}^{\gamma, k} \check{f}(\theta)=\mathfrak{g}_{\alpha, \beta}^{\gamma, k} f(-\theta)$. Using now the identities $f_{\text {even }}=(f+\check{f}) / 2$ and $f_{\text {odd }}=$ $(f-\check{f}) / 2$ we arrive at the upper estimate in (4.3). The claim follows.

Using (4.3) and then (4.1), (4.2), together with the symmetries of $\mathbb{H}_{t}^{\alpha, \beta} f_{\text {even }}$ and $\mathbb{H}_{t}^{\alpha, \beta} f_{\text {odd }}$, we obtain

$$
\begin{aligned}
\left\|\mathfrak{g}_{\alpha, \beta}^{\gamma, k} f\right\|_{L^{p}(-\pi, \pi)} & \simeq\left\|\mathfrak{g}_{\alpha, \beta}^{\gamma, k} f_{\text {even }}\right\|_{L^{p}(-\pi, \pi)}+\left\|\mathfrak{g}_{\alpha, \beta}^{\gamma, k} f_{\text {odd }}\right\|_{L^{p}(-\pi, \pi)} \\
& \simeq\left\|g_{\alpha, \beta}^{\gamma, k} f_{\text {even }}^{+}\right\|_{L^{p}(0, \pi)}+\left\|g_{\alpha+1, \beta+1}^{\gamma, k} f_{\text {odd }}^{+}\right\|_{L^{p}(0, \pi)} .
\end{aligned}
$$

This finishes the proof. 
In the remaining part of this section we deal with the case $\alpha+\beta=-1$, which is not covered by Theorem 4.2. Actually, only a slight modification is needed, and this is connected with the fact that for $\alpha+\beta=-1$ the potential spaces are defined via the Bessel type potentials $\left(\mathrm{Id}+\mathbb{L}_{\alpha, \beta}\right)^{-s / 2}$. The main idea of what follows is taken from [17, Section 4]. Here we give only a general outline and state the relevant result. The details consist of a combination of the facts and results described at the end of [17, Section 4] and the arguments already used in this section. This is left to interested readers.

Consider the modified symmetrized Jacobi 'Laplacian'

$$
\widetilde{\mathbb{L}}_{\alpha, \beta}:=\left(\operatorname{Id}+\mathbb{L}_{\alpha, \beta}^{1 / 2}\right)^{2}
$$

and the related modified Riesz type potentials $\widetilde{\mathbb{L}}_{\alpha, \beta}^{-s / 2}$. Since the spectrum of $\widetilde{\mathbb{L}}_{\alpha, \beta}$ is separated from 0 , the latter operators are well defined spectrally. Moreover, they extend to bounded operators on $L^{p}(-\pi, \pi), p \in E(\alpha, \beta)$. Since these extensions are one-to-one on $L^{p}(-\pi, \pi)$, one can define for $p \in E(\alpha, \beta)$ the modified potential spaces as

$$
\widetilde{\mathcal{L}}_{\alpha, \beta}^{p, s}(-\pi, \pi):=\widetilde{\mathbb{L}}_{\alpha, \beta}^{-s / 2}\left(L^{p}(-\pi, \pi)\right),
$$

with the norm $\|f\|_{\widetilde{\mathcal{L}}_{\alpha, \beta}^{p, s}(-\pi, \pi)}:=\|g\|_{L^{p}(-\pi, \pi)}$, where $g$ is such that $f=\widetilde{\mathbb{L}}_{\alpha, \beta}^{-s / 2} g$. These are Banach spaces, and the crucial fact is that they are isomorphic to the non-modified potential spaces $\mathcal{L}_{\alpha, \beta}^{p, s}(-\pi, \pi)$.

The Poisson semigroup related to $\widetilde{\mathbb{L}}_{\alpha, \beta}$ is generated by $-\operatorname{Id}-\mathbb{L}_{\alpha, \beta}^{1 / 2}$, hence it has the form $\left\{e^{-t} \mathbb{H}_{t}^{\alpha, \beta}\right\}$. Consequently, the relevant square function is given by

$$
\widetilde{\mathfrak{g}}_{\alpha, \beta}^{\gamma, k} f(\theta)=\left(\int_{0}^{\infty}\left|t^{k-\gamma} \frac{\partial^{k}}{\partial t^{k}}\left[e^{-t} \mathbb{H}_{t}^{\alpha, \beta} f(\theta)\right]\right|^{2} \frac{d t}{t}\right)^{1 / 2}, \quad \theta \in(-\pi, \pi) \backslash\{0\},
$$

where $0<\gamma<k$ and $k=1,2, \ldots$

The desired alternative characterization of $\mathcal{L}_{\alpha, \beta}^{p, s}(-\pi, \pi)$ reads as follows.

Theorem 4.3. Let $\alpha, \beta>-1$ and $p \in E(\alpha, \beta)$. Fix $0<\gamma<k$ with $k \in \mathbb{N}$. Then $f \in$ $\mathcal{L}_{\alpha, \beta}^{p, \gamma}(-\pi, \pi)$ if and only if $f \in L^{p}(-\pi, \pi)$ and $\tilde{\mathfrak{g}}_{\alpha, \beta}^{\gamma, k} f \in L^{p}(-\pi, \pi)$. Moreover,

$$
\|f\|_{\mathcal{L}_{\alpha, \beta}^{p, \gamma}(-\pi, \pi)} \simeq\left\|\tilde{\mathfrak{g}}_{\alpha, \beta}^{\gamma, k} f\right\|_{L^{p}(-\pi, \pi)}, \quad f \in \mathcal{L}_{\alpha, \beta}^{p, \gamma}(-\pi, \pi) .
$$

\section{$5 \quad$ Structural and embedding theorems for potential spaces}

We now show some results revealing relations between the symmetrized potential spaces with different parameters and also establishing mapping properties of certain operators with respect to the potential spaces. At the end of this section we state an analogue of the classical Sobolev embedding theorem for the symmetrized potential spaces.

The results of Section 3 suggest the following alternative definition of Riesz transforms in the symmetrized setting. For $k \geq 1$ integer, we let

$$
\mathfrak{R}_{\alpha, \beta}^{k}= \begin{cases}\mathfrak{D}_{\alpha, \beta}^{(k)} \mathbb{L}_{\alpha, \beta}^{-k / 2}, & \alpha+\beta \neq-1, \\ \mathfrak{D}_{\alpha, \beta}^{(k)}\left(\mathrm{Id}+\mathbb{L}_{\alpha, \beta}\right)^{-k / 2}, & \alpha+\beta=-1\end{cases}
$$

Notice that $\mathfrak{R}_{\alpha, \beta}^{k}$ is well defined on $\mathbb{S}_{\alpha, \beta}$. In the structural theorem below both $\mathfrak{R}_{\alpha, \beta}^{k}$ and $\mathfrak{D}_{\alpha, \beta}^{(k)}$ are understood as operators given initially on $\mathbb{S}_{\alpha, \beta}$. 
Theorem 5.1. Let $\alpha, \beta>-1$ and $p, q \in E(\alpha, \beta)$. Assume that $s, t>0$ and $k>0$ is even.

(i) If $p<q$, then $\mathcal{L}_{\alpha, \beta}^{q, s}(-\pi, \pi) \subset \mathcal{L}_{\alpha, \beta}^{p, s}(-\pi, \pi)$.

(ii) If $s<t$, then $\mathcal{L}_{\alpha, \beta}^{p, t}(-\pi, \pi) \subset \mathcal{L}_{\alpha, \beta}^{p, s}(-\pi, \pi) \subset L^{p}(-\pi, \pi)$.

Moreover, the embeddings in $(i)$ and $(i i)$ are proper and continuous.

(iii) $\mathbb{L}_{\alpha, \beta}^{-t / 2}$ establishes an isometric isomorphism between $\mathcal{L}_{\alpha, \beta}^{p, s}(-\pi, \pi)$ and $\mathcal{L}_{\alpha, \beta}^{p, s+t}(-\pi, \pi)$.

(iv) If $k<s$, then $\mathfrak{D}_{\alpha, \beta}^{(k)}$ extends to a bounded operator from $\mathcal{L}_{\alpha, \beta}^{p, s}(-\pi, \pi)$ to $\mathcal{L}_{\alpha+k, \beta+k}^{p, s-k}(-\pi, \pi)$. Moreover, $\mathfrak{D}_{\alpha, \beta}^{(k)}$ extends to a bounded operator from $\mathcal{L}_{\alpha, \beta}^{p, k}(-\pi, \pi)$ to $L^{p}(-\pi, \pi)$.

(v) $\mathfrak{R}_{\alpha, \beta}^{k}$ extends to a bounded operator from $\mathcal{L}_{\alpha, \beta}^{p, s}(-\pi, \pi)$ to $\mathcal{L}_{\alpha+k, \beta+k}^{p, s}(-\pi, \pi)$.

Proof. Throughout the proof we assume $\alpha+\beta \neq-1$. The opposite case requires analogous arguments (with (iv) and (v) requiring a little bit more attention) and is left to the reader.

To justify (i) it suffices to observe that $\|\cdot\|_{L^{p}(-\pi, \pi)}$ is controlled by $\|\cdot\|_{L^{q}(-\pi, \pi)}$ whenever $p<q$. To demonstrate (ii), let $f \in \mathcal{L}_{\alpha, \beta}^{p, t}(-\pi, \pi)$. Then there exists $g \in L^{p}(-\pi, \pi)$ such that $f=\mathbb{L}_{\alpha, \beta}^{-t / 2} g$, so by Proposition 2.1

$$
f=\mathbb{L}_{\alpha, \beta}^{-t / 2} g=\mathbb{L}_{\alpha, \beta}^{-s / 2} \mathbb{L}_{\alpha, \beta}^{-(t-s) / 2} g \in \mathcal{L}_{\alpha, \beta}^{p, s}(-\pi, \pi) .
$$

Here second identity is straightforward for $g \in \mathbb{S}_{\alpha, \beta}$, and for $g \in L^{p}(-\pi, \pi)$ it follows by Proposition 2.1 and an approximation argument.

In view of the above, the inclusions in (i) and (ii) are continuous. To see that the inclusion in (i) is proper it suffices to take $f=\mathbb{L}_{\alpha, \beta}^{-s / 2} g$ with some $g \in L^{p}(-\pi, \pi) \backslash L^{q}(-\pi, \pi)$. Then obviously $f \in \mathcal{L}_{\alpha, \beta}^{p, s}(-\pi, \pi)$. On the other hand, $f \notin \mathcal{L}_{\alpha, \beta}^{q, s}(-\pi, \pi)$. Indeed, otherwise there would exist $\widetilde{g} \in L^{q}(-\pi, \pi)$ such that $f=\mathbb{L}_{\alpha, \beta}^{-s / 2} \widetilde{g}$, a contradiction with injectivity of $\mathbb{L}_{\alpha, \beta}^{-s / 2}$ on $L^{p}(-\pi, \pi)$, see Proposition 2.4. To prove that the inclusions in (ii) are proper we first recall that an analogous result holds in the non-symmetrized setting, see [17, Theorem 3.1(i)]. This means that there exist $h_{1} \in \mathcal{L}_{\alpha, \beta}^{p, s}(0, \pi) \backslash \mathcal{L}_{\alpha, \beta}^{p, t}(0, \pi)$ and $h_{2} \in L^{p}(0, \pi) \backslash \mathcal{L}_{\alpha, \beta}^{p, s}(0, \pi)$. Let $f_{1}$ and $f_{2}$ be even extensions of $h_{1}$ and $h_{2}$, respectively, to $(-\pi, \pi)$. Then Proposition 2.5 implies $f_{1} \in \mathcal{L}_{\alpha, \beta}^{p, s}(-\pi, \pi) \backslash \mathcal{L}_{\alpha, \beta}^{p, t}(-\pi, \pi)$ and $f_{2} \in L^{p}(-\pi, \pi) \backslash \mathcal{L}_{\alpha, \beta}^{p, s}(-\pi, \pi)$.

To show that (iii) holds, it suffices to observe that, see (5.1),

$$
\mathbb{L}_{\alpha, \beta}^{-t / 2} \mathbb{L}_{\alpha, \beta}^{-s / 2} g=\mathbb{L}_{\alpha, \beta}^{-(t+s) / 2} g, \quad g \in L^{p}(-\pi, \pi) .
$$

Proving (iv) is reduced to showing the bound

$$
\left\|\mathbb{L}_{\alpha+k, \beta+k}^{(s-k) / 2} \mathfrak{D}_{\alpha, \beta}^{(k)} \mathbb{L}_{\alpha, \beta}^{-s / 2} g\right\|_{L^{p}(-\pi, \pi)} \lesssim\|g\|_{L^{p}(-\pi, \pi)}, \quad g \in \mathbb{S}_{\alpha, \beta} .
$$

Since $k$ is even, $\mathfrak{d}_{\alpha, \beta}^{(k)} g_{\text {even }}$ and $\mathfrak{d}_{\alpha+1, \beta+1}^{(k)} g_{\text {odd }}$ are even and odd functions, respectively. Therefore, in view of $(2.3)$,

$$
\mathbb{L}_{\alpha+k, \beta+k}^{(s-k) / 2} \mathfrak{D}_{\alpha, \beta}^{(k)} \mathbb{L}_{\alpha, \beta}^{-s / 2} g=\left(\mathbb{L}_{\alpha+k, \beta+k}^{(s-k) / 2}\right)_{\mathrm{e}} \mathfrak{d}_{\alpha, \beta}^{(k)}\left(\mathbb{L}_{\alpha, \beta}^{-s / 2}\right)_{\mathrm{e}} g_{\text {even }}+\left(\mathbb{L}_{\alpha+k, \beta+k}^{(s-k) / 2}\right)_{\mathrm{o}} \mathfrak{d}_{\alpha+1, \beta+1}^{(k)}\left(\mathbb{L}_{\alpha, \beta}^{-s / 2}\right)_{\mathrm{o}} g_{\text {odd }} .
$$

Now recall that in the non-symmetrized setting, see [17, Theorem 3.1(iii)], we have

$$
\left\|L_{\alpha+k, \beta+k}^{(s-k) / 2} \mathfrak{d}_{\alpha, \beta}^{(k)} L_{\alpha, \beta}^{-s / 2} h\right\|_{L^{p}(0, \pi)} \lesssim\|h\|_{L^{p}(0, \pi)}, \quad h \in S_{\alpha, \beta} .
$$

Using this bound and its variant with $\alpha$ and $\beta$ replaced by $\alpha+1$ and $\beta+1$, respectively, we obtain

$$
\left\|\mathbb{L}_{\alpha+k, \beta+k}^{(s-k) / 2} \mathfrak{D}_{\alpha, \beta}^{(k)} \mathbb{L}_{\alpha, \beta}^{-s / 2} g\right\|_{L^{p}(-\pi, \pi)}
$$




$$
\begin{aligned}
& \simeq\left\|\left(\mathbb{L}_{\alpha+k, \beta+k}^{(s-k) / 2}\right)_{\mathrm{e}} \mathfrak{d}_{\alpha, \beta}^{(k)}\left(\mathbb{L}_{\alpha, \beta}^{-s / 2}\right)_{\mathrm{e}} g_{\text {even }}\right\|_{L^{p}(-\pi, \pi)}+\left\|\left(\mathbb{L}_{\alpha+k, \beta+k}^{(s-k) / 2}\right)_{\mathrm{o}} \mathfrak{d}_{\alpha+1, \beta+1}^{(k)}\left(\mathbb{L}_{\alpha, \beta}^{-s / 2}\right)_{\mathrm{o}} g_{\text {odd }}\right\|_{L^{p}(-\pi, \pi)} \\
& \simeq\left\|L_{\alpha+k, \beta+k}^{(s-k) / 2} \mathfrak{d}_{\alpha, \beta}^{(k)} L_{\alpha, \beta}^{-s / 2} g_{\text {even }}^{+}\right\|_{L^{p}(0, \pi)}+\left\|L_{\alpha+1+k, \beta+1+k}^{(s-k) / 2} \mathfrak{d}_{\alpha+1, \beta+1}^{(k)} L_{\alpha+1, \beta+1}^{-s / 2} g_{\text {odd }}^{+}\right\|_{L^{p}(0, \pi)} \\
& \lesssim\left\|g_{\text {even }}^{+}\right\|_{L^{p}(0, \pi)}+\left\|g_{\text {odd }}^{+}\right\|_{L^{p}(0, \pi)} \simeq\|g\|_{L^{p}(-\pi, \pi)},
\end{aligned}
$$

as required.

Finally, (v) is a consequence of (iv) and the boundedness of $\mathbb{L}_{\alpha, \beta}^{-k / 2}$ from $\mathcal{L}_{\alpha, \beta}^{p, s}(-\pi, \pi)$ to $\mathcal{L}_{\alpha, \beta}^{p, s+k}(-\pi, \pi)$, see (iii).

Parts (iv) and (v) of Theorem 5.1, as stated, do not hold when $k$ is an odd number. Roughly speaking, this is because in such a case $\mathfrak{D}_{\alpha, \beta}^{(k)}$ switches symmetry of functions from even to odd and vice versa. However, a slight modification of $\mathfrak{D}_{\alpha, \beta}^{(k)}$ makes the statements (iv) and (v) true for all $k \geq 1$. Indeed, one easily verifies that the arguments proving (iv) and (v) go through with $\mathfrak{D}_{\alpha, \beta}^{(k)}$ replaced by $\widetilde{\mathfrak{D}}_{\alpha, \beta}^{(k)}$ defined by $\widetilde{\mathfrak{D}}_{\alpha, \beta}^{(k)} f(\theta)=\operatorname{sign}^{k}(\theta) \mathfrak{D}_{\alpha, \beta}^{(k)} f(\theta)$.

Another interesting question is whether there are any inclusions between potential spaces with different parameters of type. It turns out that in general the answer is negative.

Proposition 5.2. Let $\alpha, \beta, \gamma, \delta>-1$ be such that $(\alpha, \beta) \neq(\gamma, \delta)$. Assume that $p \in E(\alpha, \beta) \cap$ $E(\gamma, \delta)$ and $\alpha, \beta, \gamma, \delta \leq-1 / p+1 / 2$. Then neither the inclusion $\mathcal{L}_{\alpha, \beta}^{p, 1}(-\pi, \pi) \subset \mathcal{L}_{\gamma, \delta}^{p, 1}(-\pi, \pi)$ nor the inclusion $\mathcal{L}_{\gamma, \delta}^{p, 1}(-\pi, \pi) \subset \mathcal{L}_{\alpha, \beta}^{p, 1}(-\pi, \pi)$ holds.

Proof. Take $f_{1}=\Psi^{\alpha, \beta}$ and $f_{2}=\Psi^{\gamma, \delta}$. With the aid of [16, formula (8)] one verifies that $f_{1}^{+}, f_{2}^{+}$, $D_{\alpha, \beta} f_{1}^{+}, D_{\gamma, \delta} f_{2}^{+} \in L^{p}(0, \pi)$, but $D_{\alpha, \beta} f_{2}^{+}, D_{\gamma, \delta} f_{1}^{+} \notin L^{p}(0, \pi)$. Thus $f_{1}^{+} \in W_{\alpha, \beta}^{p, 1}(0, \pi) \backslash W_{\gamma, \delta}^{p, 1}(0, \pi)$ and $f_{2}^{+} \in W_{\gamma, \delta}^{p, 1}(0, \pi) \backslash W_{\alpha, \beta}^{p, 1}(0, \pi)$. Since $f_{1}$ and $f_{2}$ are even functions, it follows by Proposition 3.1 that $f_{1} \in \mathcal{L}_{\alpha, \beta}^{p, 1}(-\pi, \pi) \backslash \mathcal{L}_{\gamma, \delta}^{p, 1}(-\pi, \pi)$ and $f_{2} \in \mathcal{L}_{\gamma, \delta}^{p, 1}(-\pi, \pi) \backslash \mathcal{L}_{\alpha, \beta}^{p, 1}(-\pi, \pi)$.

We finish this section with a counterpart of the classical Sobolev embedding theorem. The statement below is a direct consequence of an analogous result in the non-symmetrized situation [17, Theorem 3.2] and Proposition 2.5. We leave the details to the interested readers.

Theorem 5.3. Let $\alpha, \beta>-1, p \in E(\alpha, \beta)$ and $1 \leq q<p(\alpha, \beta)$.

(i) If $s>0$ is such that $1 / q \geq 1 / p-s$, then $\mathcal{L}_{\alpha, \beta}^{p, s}(-\pi, \pi) \subset L^{q}(-\pi, \pi)$ and

$$
\|f\|_{q} \lesssim\|f\|_{\mathcal{L}_{\alpha, \beta}^{p, s}(-\pi, \pi)}, \quad f \in \mathcal{L}_{\alpha, \beta}^{p, s}(-\pi, \pi)
$$

(ii) If $\alpha, \beta \geq-1 / 2$ and $s>1 / p$, then $\mathcal{L}_{\alpha, \beta}^{p, s}(-\pi, \pi) \subset C(-\pi, \pi)$ and (5.2) holds with $q=\infty$.

\section{Sample applications of potential spaces}

The study of symmetrized potential spaces performed in the previous sections reveals that the symmetrized objects inherit many of the properties of their non-symmetrized prototypes. Furthermore, most of the proofs were based on the arguments relying on suitable decompositions of the operators into their even and odd symmetric parts. This actually reduced our problems to the non-symmetrized setup. Therefore, it comes as no surprise that both theories, symmetrized and non-symmetrized, have parallel applications. Below we present some results which illustrate the utility of the symmetrized potential spaces. The proofs combine the symmetry arguments that have already appeared in this paper with the results from [17, Section 5]. We leave them to the reader. 
Given some initial data $f \in L^{2}(-\pi, \pi)$, consider the following Cauchy problem based on the symmetrized Jacobi operator:

$$
\left\{\begin{array}{l}
\left(i \partial_{t}+\mathbb{L}_{\alpha, \beta}\right) u(\theta, t)=0, \\
u(\theta, 0)=f(\theta),
\end{array} \quad \text { a.a. } \theta \in(-\pi, \pi), \quad t \in \mathbb{R} .\right.
$$

Then $\exp \left(i t \mathbb{L}_{\alpha, \beta}\right) f$, understood spectrally, is a solution to this problem. The next result shows that the theory of symmetrized Jacobi potential spaces can be used to study pointwise almost everywhere convergence of this solution to the initial condition.

Proposition 6.1. Let $\alpha, \beta>-1$ and $s>1 / 2$. Then for each $f \in \mathcal{L}_{\alpha, \beta}^{2, s}(-\pi, \pi)$

$$
\lim _{t \rightarrow 0} \exp \left(i t \mathbb{L}_{\alpha, \beta}\right) f(\theta)=f(\theta) \quad \text { a.a. } \theta \in(-\pi, \pi) .
$$

Furthermore, one can estimate a mixed norm of the solution in terms of the potential norm of the initial condition.

Proposition 6.2. Let $\alpha, \beta>-1$ and $p \in E(\alpha, \beta)$. Assume that $q \geq 2$ and $s>0$ is such that $s \geq 3 / 2+\max \{\alpha, \beta\}$ and $\alpha+\beta$ is integer. Then

$$
\left\|\exp \left(i t \mathbb{L}_{\alpha, \beta}\right) f\right\|_{L_{\theta}^{p}\left((-\pi, \pi), L_{t}^{q}(0,2 \pi)\right)} \lesssim\|f\|_{\mathcal{L}_{\alpha, \beta}^{2, s+1-2 / q}(-\pi, \pi)}, \quad f \in \mathcal{L}_{\alpha, \beta}^{2, s+1-2 / q}(-\pi, \pi) .
$$

\section{Acknowledgment}

The author would like to express his gratitude to Professor Adam Nowak for indicating the topic and constant support during the preparation of this paper. Research supported by the National Science Centre of Poland, project No. 2013/09/N/ST1/04120.

\section{References}

[1] Almeida V., Betancor J.J., Castro A.J., Sanabria A., Scotto R., Variable exponent Sobolev spaces associated with Jacobi expansions, arXiv:1410.3642.

[2] Balderrama C., Urbina R. W.O., Fractional integration and fractional differentiation for Jacobi expansions, Divulg. Mat. 15 (2007), 93-113.

[3] Balderrama C., Urbina R. W.O., Fractional integration and fractional differentiation for $d$-dimensional Jacobi expansions, in Special Functions and Orthogonal Polynomials, Contemp. Math., Vol. 471, Amer. Math. Soc., Providence, RI, 2008, 1-14, math.AP/0608639.

[4] Betancor J.J., Fariña J.C., Rodríguez-Mesa L., Testoni R., Torrea J.L., A choice of Sobolev spaces associated with ultraspherical expansions, Publ. Mat. 54 (2010), 221-242.

[5] Betancor J.J., Fariña J.C., Rodríguez-Mesa L., Testoni R., Torrea J.L., Fractional square functions and potential spaces, J. Math. Anal. Appl. 386 (2012), 487-504.

[6] Bongioanni B., Torrea J.L., Sobolev spaces associated to the harmonic oscillator, Proc. Indian Acad. Sci. Math. Sci. 116 (2006), 337-360, math.CO/0608684.

[7] Bongioanni B., Torrea J.L., What is a Sobolev space for the Laguerre function systems?, Studia Math. 192 (2009), 147-172.

[8] Calderón C.P., Urbina R. W.O., On Abel summability of Jacobi polynomials series, the Watson kernel and applications, Illinois J. Math. 57 (2013), 343-371, arXiv:1207.4524.

[9] Castro A.J., Nowak A., Szarek T.Z., Riesz-Jacobi transforms as principal value integrals, J. Fourier Anal. Appl., to appear, arXiv:1405.7069.

[10] Chouchene F., Harmonic analysis associated with the Jacobi-Dunkl operator on $]-\frac{\pi}{2}, \frac{\pi}{2}[$, J. Comput. Appl. Math. 178 (2005), 75-89. 
[11] Ciaurri Ó., Roncal L., Stinga P.R., Fractional integrals on compact Riemannian symmetric spaces of rank one, Adv. Math. 235 (2013), 627-647, arXiv:1205.3957.

[12] Ciaurri Ó., Roncal L., Stinga P.R., Riesz transforms on compact Riemannian symmetric spaces of rank one, Milan J. Math., to appear, arXiv:1308.6507.

[13] Graczyk P., Loeb J.J., López P. I.A., Nowak A., Urbina R. W.O., Higher order Riesz transforms, fractional derivatives, and Sobolev spaces for Laguerre expansions, J. Math. Pures Appl. 84 (2005), 375-405.

[14] Koornwinder T.H., Bouzeffour F., Non-symmetric Askey-Wilson polynomials as vector-valued polynomials, Appl. Anal. 90 (2011), 731-746, arXiv:1006.1140.

[15] Langowski B., Harmonic analysis operators related to symmetrized Jacobi expansions, Acta Math. Hungar. 140 (2013), 248-292, arXiv:1210.1342.

[16] Langowski B., Sobolev spaces associated with Jacobi expansions, J. Math. Anal. Appl. 420 (2014), 15331551, arXiv:1312.7285.

[17] Langowski B., On potential spaces related to Jacobi expansions, J. Math. Anal. Appl. 432 (2015), 374-397, arXiv:1410.6635.

[18] Muckenhoupt B., Transplantation theorems and multiplier theorems for Jacobi series, Mem. Amer. Math. Soc. 64 (1986), iv+86 pages.

[19] Nowak A., Roncal L., Potential operators associated with Jacobi and Fourier-Bessel expansions, J. Math. Anal. Appl. 422 (2015), 148-184, arXiv:1212.6342.

[20] Nowak A., Sjögren P., Riesz transforms for Jacobi expansions, J. Anal. Math. 104 (2008), 341-369, arXiv:0802.2369.

[21] Nowak A., Sjögren P., Calderón-Zygmund operators related to Jacobi expansions, J. Fourier Anal. Appl. 18 (2012), 717-749, arXiv:1011.3615.

[22] Nowak A., Sjögren P., Sharp estimates of the Jacobi heat kernel, Studia Math. 218 (2013), 219-244, arXiv:1111.3145.

[23] Nowak A., Sjögren P., Szarek T.Z., Analysis related to all admissible type parameters in the Jacobi setting, Constr. Approx. 41 (2015), 185-218, arXiv:1211.3270.

[24] Nowak A., Stempak K., $L^{2}$-theory of Riesz transforms for orthogonal expansions, J. Fourier Anal. Appl. 12 (2006), 675-711.

[25] Nowak A., Stempak K., A symmetrized conjugacy scheme for orthogonal expansions, Proc. Roy. Soc. Edinburgh Sect. A 143 (2013), 427-443, arXiv:1009.1767.

[26] Radha R., Thangavelu S., Multipliers for Hermite and Laguerre Sobolev spaces, J. Anal. 12 (2004), $183-191$.

[27] Segovia C., Wheeden R.L., On certain fractional area integrals, J. Math. Mech. 19 (1970), 247-262.

[28] Stempak K., Jacobi conjugate expansions, Studia Sci. Math. Hungar. 44 (2007), 117-130.

[29] Szegő G., Orthogonal polynomials, American Mathematical Society, Colloquium Publications, Vol. 23, 4th ed., Amer. Math. Soc., Providence, R.I., 1975. 